\title{
Módulos Alternativos en la Enseñanza de las Ciencias. Estrategia Didáctica Orientada al Logro de Aprendizajes Significativos
}

\author{
Sonia E. Oses ${ }^{(1)}$ y Laura E. Carrasco ${ }^{(2)}$ * \\ Universidad de la Frontera, (1) Facultad de Educación, Ciencias Sociales y Humanidades, Departamento de \\ Educación, (2) Facultad de Medicina, Departamento de Ciencias Básicas, Casilla 54-D, Temuco-Chile \\ (e-mail: sonia.osses@ufrontera.cl; laura.carrasco@ufrontera.cl) \\ * Autor a quien debe ser dirigida la correspondencia
}

Recibido Feb. 22, 2013; Aceptado Mar. 12, 2013; Versión final recibida Abr. 09, 2013

Resumen

Se presenta un estudio sobre la estrategia didáctica denominada Módulos Alternativos de Aprendizaje, basada en el enfoque constructivista del aprendizaje y que está orientada a favorecer el logro de aprendizajes significativos. El objetivo del estudio es caracterizar los módulos y ejemplificarlos a través del tema Drogas y Toxicomanía aplicando la metodología a estudiantes de Segundo Ciclo de Educación Media de comunas vulnerables de la región de la Araucanía en Chile. Se discute dichos resultados y se concluye que los Módulos Alternativos de Aprendizaje han contribuido al desarrollo de conocimientos en profundidad, a mejorar la capacidad de reflexionar sobre la forma en que aprenden los estudiantes, a la mejor autorregulación de su propio proceso de aprendizaje y a la práctica de valores a nivel personal y social.

Palabras clave: módulos alternativos, enfoque constructivista, estrategia didáctica, aprendizaje significativo

\section{Alternative Modules in Science Education. Didactic Strategy to Achieve Meaningful Learning}

\begin{abstract}
This paper presents a study on the didactic strategy called Alternative Learning Modules, based on the constructive approach of learning and aimed to contribute in achieving meaningful learning. The objective of the study is to characterize the modules, exemplifying them with the subject Drugs and Drug Addiction applying the methodology to students of the Second Cycle in High-school of vulnerable towns of the Region of the Araucania in Chile. The results are discussed concluding that the Alternative Learning Modules have contributed to develop knowledge in depth, to improve the capacity of good thinking about the way in which students learn, to self-regulating their own learning process and to practice of personal and social values.
\end{abstract}

Keywords: alternative modules, constructive approach, didactic strategy, meaningful learning 


\section{INTRODUCCIÓN}

Según Ontoria et al. (2003), aprender es una experiencia personal que permite construir conocimiento, en la cual interviene el yo con todas sus capacidades, emociones, sentimientos, motivaciones y habilidades. En este contexto, a diferencia de la enseñanza tradicional - que centra el aprendizaje en el profesor transmisor y los estudiantes desempeñan un rol predominantemente memorístico y pasivo - el profesor es un mediador del aprendizaje y el alumno, un constructor activo de su propio aprendizaje. Correa (2004), destaca la importancia del aprendizaje significativo dentro del enfoque constructivista del aprendizaje, ya que aquel contribuye a generar estructuras cognitivas que permiten al aprendiz relacionar la nueva información con los conocimientos previos, produciéndose la transformación cualitativa de éstos, de modo que alcanzan mayor complejidad y profundidad, como asimismo, una retención más duradera y proyectable a la resolución de problemas. Desde este enfoque, Pimienta (2008) considera que la educación es un proceso en el cual aprender equivale a construir conocimientos propios y enseñar significa construir con una actitud mediadora al logro de esa construcción. Al respecto, Shulman (2005), para mejorar el proceso pedagógico, presenta un modelo de razonamiento y acción pedagógica que incluye: la comprensión de los objetivos, la transformación del aprendizaje para interpretar y analizar críticamente los textos de estudio, el uso de representaciones, la selección de material, la adaptación a las características de los alumnos, la enseñanza activa, el trabajo grupal, la autoevaluación, la coevaluación y la reflexión.

En la línea de este nuevo enfoque, el Proyecto FONDECYT Regular $\mathrm{N}^{\circ} 1100378$, de 3 años de duración (Osses, 2010) - que, desde la perspectiva epistemológica corresponde al paradigma cualitativo en el ámbito de la Enseñanza de las Ciencias - apunta al desarrollo de capacidades y valores implícitos en un aprendizaje de calidad, a través de adecuadas estrategias de aprendizaje por parte de los alumnos de Segundo Ciclo de Enseñanza Media de cuatro Comunas vulnerables de la Región de la Araucanía, y de adecuadas estrategias de enseñanza por parte de sus profesores de Biología. Desde el punto de vista metodológico, corresponde a una investigación mixta, dado que se utilizó técnicas de recolección de datos cuantitativas y cualitativas. Entre las técnicas cuantitativas se aplicó un instrumento para medir metacognición (Escala tipo Likert) antes de comenzar a aplicar los módulos y después de finalizados. Los resultados obtenidos a través de este instrumento, mostraron claramente los avances realizados por los estudiantes en su aprendizaje, específicamente, en el ámbito metacognitivo (Osses, 2013).

Entre las técnicas cualitativas utilizadas, se puede mencionar: observación participante, entrevistas en profundidad y grupos focales. En coherencia con la naturaleza cualitativa de la investigación, (Hennink et al., 2011) no se plantean hipótesis sino supuestos, no se aplican tests estadísticos sino técnicas cualitativas de análisis de datos y no se plantean conclusiones sino que se mencionan los hallazgos. Este artículo correspondiente al ámbito cualitativo - se refiere al supuesto: "es posible lograr un avance en la calidad de los aprendizajes logrados por los estudiantes y construir conocimiento sobre ello, mediante la incorporación en la práctica educativa, por parte del profesor, de estrategias de enseñanza claramente especificadas y orientadas a generar conocimiento, adecuada selección y manejo de estrategias de aprendizaje cognitivas y afectivas por parte de los estudiantes". Se trata de una Investigación-Acción (Álvarez-Gayou, 2005), de primer orden por parte de los docentes de las Comunas seleccionadas, directamente involucrados en el logro de los aprendizajes de calidad por parte de los estudiantes y de una Investigación-Acción de segundo orden por parte de los investigadores, quienes apoyaron el proceso de desarrollo de dicho aprendizaje en los alumnos y han construido conocimiento sobre él. Los sujetos participantes en la investigación corresponden a estudiantes de Tercero y Cuarto año medio de los Liceos Municipalizados de las Comunas: Puerto Saavedra, Carahue, Lonquimay y Collipulli y sus profesores de Biología, con un promedio anual de 150 alumnos en Tercero, 120 alumnos en Cuarto y cuatro profesores en total, es decir, uno por comuna. Desde la perspectiva pedagógica, los Módulos Alternativos de Aprendizaje en Biología, que prepararon los profesores y utilizaron los estudiantes, incorporan las estrategias cognitivas y afectivas en el desarrollo de los contenidos propios de la asignatura.

La recolección de la información, se organizó en torno a ciclos de Investigación-Acción, para lo cual se utilizó técnicas tales como: observación participante, entrevistas en profundidad y grupos focales (Robledo, 2009; Bautista, 2011). Se realizó: 42 observaciones de clases y 12 entrevistas en profundidad a los profesores, por parte de los investigadores durante la puesta en práctica de los Módulos; 3 grupos focales a los profesores, antes de iniciar el desarrollo de los Módulos y 3 después de finalizados y 16 grupos focales a los estudiantes, una vez terminada la aplicación de los Módulos. Los datos obtenidos fueron analizados a través del programa Atlas ti. En el análisis de datos, se sigue el esquema propuesto por Miles y Huberman (1994) que distingue: reducción de datos, disposición de datos y planteamiento de conclusiones. Para verificar la validez de la información, se aplicó las técnicas de triangulación y saturación de datos (Pérez, 1994). Por su naturaleza cualitativa, los resultados de la investigación no pueden extrapolarse más allá de los sujetos participantes en la investigación. 


\section{MÓDULOS DE APRENDIZAJE ALTERNATIVOS}

La utilización de la Estrategia Modular denominada Alternativa, para distinguirla de aquella de origen conductista, presenta varias ventajas de tipo pedagógico, tales como: promover la flexibilidad en su uso y la actividad de los estudiantes, favorecer la cooperación e intercambio de experiencias intra e inter grupos y respetar las diferencias y los ritmos de avance individuales, todo lo cual, permite al profesor asumir su rol educativo como mediador del proceso.

En los Módulos de Aprendizaje Alternativos (Anexo $\mathrm{N}^{\circ} 1$ ), se comienza por detectar los conocimientos previos, es decir, aquellos conocimientos que los estudiantes traen y confirmarán o transformarán durante el proceso educativo. Desde el punto de vista del enfoque constructivista del aprendizaje, esta etapa es fundamental, porque permite al profesor establecer relación con la realidad de los alumnos, favoreciendo el logro de aprendizajes significativos. En este contexto, Mahmud y Gutiérrez (2010) utilizando una estrategia de enseñanza basada en el cambio conceptual para transformar las ideas previas de los alumnos universitarios, lograron mejorar su comprensión en ciencias físicas. En el mismo ámbito, Bogumil y Abad (2008) alcanzaron avances en el aprendizaje y rendimiento de grupos reducidos de estudiantes del área de Ingeniería.

Para que los Módulos de Aprendizaje Alternativos permitan lograr en plenitud los objetivos planteados, se requiere que cumplan ciertas condiciones, tales como: a) estar impregnados del enfoque constructivista del aprendizaje; b) estructurar el material de aprendizaje de modo tal que los módulos incluyan tópicos pertinentes $y$, dentro de lo posible, vivenciales para los estudiantes; c) ser equilibrados, es decir, que contengan aspectos relativos a los ámbitos cognitivo y valórico; d) que promuevan el tratamiento integrado de conceptos y procesos, ya que la psicología cognitiva actualmente cuestiona que los estudiantes aprendan las habilidades independientemente de los conceptos; e) establecer la vinculación de la ciencia con el entorno natural y sociocultural (Osses, 2002). En la elaboración de los módulos, como se señala a continuación, se ha tenido en cuenta los componentes básicos del currículo: objetivos, contenidos, estrategias didácticas y evaluación.

Los Objetivos generales de los módulos para Tercero y Cuarto año de Enseñanza Media (Osses, 1997) fueron:

- Desarrollar la capacidad de describir hechos y de comprender cómo se generan los conceptos, principios y teorías científicas que forman parte del cuerpo de conocimiento básico de la Biología.

- Desarrollar habilidades prácticas, intelectuales y de comunicación generalmente aceptadas por la comunidad científica.

- Desarrollar la comprensión de la ciencia y sus procesos en contextos diferentes (ciencia pura, ciencia aplicada, actividad cultural).

- Desarrollar actitudes positivas hacia las implicancia ambientales de la ciencia en el medio socio-.cultural y el medio natural

Contenidos: Los módulos se refieren a grandes temáticas biológicas respetando la forma especializada de visualizar los fenómenos, propia de la disciplina., lo que no impide dar, al mismo tiempo, oportunidad a los estudiantes para aproximarse a problemáticas reales desde distintas disciplinas pero en torno a objetivos comunes, tales como: lenguaje, escritura, interacción social, organización de salidas a terreno, efectos de la acción antrópica sobre el medio ambiente, entre otras. En los Módulos de Tercer Año Medio los contenidos se refirieron, específicamente, a Drogas y Toxicomanía y Estrés. En Cuarto Año Medio, en cambio, los contenidos fueron: Microbios y Sistemas de Defensa.

Las estrategias didácticas, en términos de actividades, entendidas como la integración de distintos elementos en una forma de acción a través de la cual se promueve el aprendizaje de los alumnos, consideran la realidad concreta del alumno, su entorno socio-cultural, su vida familiar, sus aspiraciones y valores personales y de su grupo de pertenencia. En los Módulos se considera la explicitación de ideas por parte de los estudiantes en forma individual, grupal o conjunta, actividades del alumnos solo, con carácter de reflexión personal que puede comunicar en forma oral o escrita, discusiones, trabajos prácticos o visitas a distintas instancias extra-aula con compañeros en grupos pequeños, puestas en común y salidas a terreno del grupo curso y profesor para estar en contacto directo con el problema en estudio y conversaciones con las familias sobre tópicos relacionados con la temática abordada. Durante el transcurso de las actividades, el docente desempeña el rol de guía del aprendizaje promoviendo la construcción o reconstrucción de conocimientos por parte de los estudiantes. A fin de que la metacognición y el trabajo grupal - que en estos Módulos son objeto de especial atención - se incorporen realmente al proceso educativo, se explicita su presencia en cada una de las Guías de Aprendizaje que conforma el Módulo: al inicio del proceso a través de los objetivos, durante el proceso, en las actividades de aprendizaje y al final, 
en la evaluación del proceso realizado.A objeto de clarificar el aporte de la metacognición y del trabajo grupal en la estrategia modular, a continuación se plantean algunas ideas sobre ambos.

Metacognición. En su definición de metacognición, Flavell (1976) citado por Mateos (2001), la identifica, por una parte, con el conocimiento de la actividad cognitiva y, por otra, con el control que se ejerce sobre la propia actividad cognitiva.

a) El conocimiento metacognitivo es el conocimiento sobre el conocimiento e implica la reflexión sobre los propios procesos cognitivos. El Mismo autor afirma que, se puede desarrollar conocimiento metacognitivo sobre tres aspectos de la actividad cognitiva: la persona, la tarea y las estrategias. El primer aspecto comprende el conocimiento y las creencias sobre las características de las personas - intraindividuales, interindividuales y universales - que son importantes para las tareas que demandan algún tipo de actividad cognitiva. Las variables intraindividuales se refieren a las propias habilidades, recursos y experiencia en la realización de diversas tareas cognitivas, intereses, motivaciones y otros atributos y estados personales que pueden afectar el rendimiento. El segundo tipo de conocimiento metacognitivo se refiere al conocimiento sobre la influencia que ejercen la naturaleza y las demandas de la tarea sobre la dificultad en su ejecución. El último tipo de conocimiento metacognitivo es el conocimiento de la efectividad de las diversas estrategias para abordar la tarea. Se diferencian dos tipos de estrategias: cognitivas y metacognitivas. Las estrategias cognitivas se emplean para hacer avanzar la actividad cognitiva hacia la meta y las metacognitivas, para supervisar ese avance. Las estrategias cognitivas se refieren al qué (contenido), al cómo (métodos y procesos) y al para qué (capacidades y valores) y posicionan la educación en el marco de la sociedad del conocimiento desde un nuevo paradigma, de modo que el curriculum y la educación constituyen una forma de intervención en procesos cognitivos y afectivos (Román, 2005). En sus estudios sobre el rol de la metacognición en las interacciones sociales, Frith (2012) sugiere que se trata de una habilidad exclusivamente humana que se desarrolla a través del perfeccionamiento en los acuerdos interpersonales.

b) En el control o autorregulación metacognitiva, se distinguen tres procesos: planificación, supervisión y evaluación, que se ponen en marcha antes, durante y después de la ejecución de una tarea, respectivamente. En investigaciones en la línea de Enseñanza de las Ciencias, Osses $(1997,2003)$ ha demostrado que, a través de Módulos de Aprendizaje, se logra motivar a los estudiantes para que confirmen o modifiquen sus conocimientos previos, trabajando en forma individual, grupal o colectiva. De este modo, ellos se convierten en protagonistas de su propio aprendizaje, para lo cual, utilizando conjuntamente su pensamiento crítico y creativo, autorregulan su propio proceso de aprendizaje a través de la reflexión orientada a la acción. Resultados de estudios efectuados sobre la dimensión metacognitiva como estrategia de aprendizaje en Biología (Jaramillo, 2009), muestran, asimismo, a la metacognición como un camino que conduce a la autonomía en el aprendizaje de los estudiantes, generando en ellos, mayores oportunidades de inserción social. Señalan, además que, a través de la elaboración y puesta en práctica de Módulos de Aprendizaje en Biología para Tercero y Cuarto Año de Enseñanza Media en Comunas vulnerables de la región de la Araucanía, es posible avanzar en niveles de autoconocimiento y autorregulación, a condición de que dichos módulos cumplan con los requisitos señalados anteriormente. Por otro lado, Hugo (2009), afirman que la práctica consciente de estrategias metacognitivas facilita el camino hacia la toma de decisiones correctas, el buen uso de su libertad y, por ende, su desarrollo integral como personas.

El trabajo en equipo. Para mejorar la capacidad de resolver problemas, no basta con el trabajo individual, sino que éste debe ser distribuido entre varias personas; la idea del trabajo en equipo se ha visto impulsada por las nuevas tecnologías, especialmente Internet, ya que hace posible acceder a conocimientos y contactos con profesores distantes geográficamente y formar parte de comunidades virtuales, lo que amplía las posibilidades en esta área (Marcelo, 2003). Al respecto Álvarez (2008) utilizando tecnologías de la información y la comunicación (TIC), a través de talleres supervisados y trabajos en grupos multidisciplinarios con alumnos del área de ingeniería y educación secundaria, logró resultados favorables, por lo cual recomienda integrar en el currículo este tipo de estrategias de aprendizaje, ya que permiten desarrollar trabajo autónomo y significativo en los estudiantes.

Al trabajar en equipo los estudiantes se ayudan, comparten habilidades, aportan, cooperan, escuchan y pueden llegar a acuerdos y lograr un clima de confianza, libertad y cordialidad. En este contexto, el profesor debe ser un intermediario, un amplificador, un adaptador, un organizador, un diseñador de procesos formativos, un guía o asesor que facilita el trabajo autónomo de los estudiantes, demostrando pasión por su labor docente, sintiéndose comprometido y motivado. Además, debe mantener vigente su desarrollo personal y profesional, preocuparse del aprendizaje de sus alumnos, manifestando actitudes favorables hacia el proceso metacognitivo, procurando que los alumnos se responsabilicen del control de su propio aprendizaje (Day, 2006). Conjuntamente con lo anterior, ha de ser un investigador en el aula, creando un ambiente de trabajo cooperativo, en el cual se logre aprender a aprender y se desarrollen actitudes y valores orientados a la crítica reflexiva y a la toma de decisiones, habida cuenta que, en un aula en que se 
estimule la creatividad, estarán también siempre presente valores como la libertad y la responsabilidad (Menchén, 2008).

Al respecto, Osuna y Luna (2011), al realizar un análisis de contenido bajo el enfoque cualitativo para identificar valores como la honestidad, responsabilidad y respeto, sugirieron estrategias metodológicas para promover dichos valores en estudiantes de Ciencias Naturales e Ingeniería y Tecnología, las cuales permiten formar personas aptas para un buen desempeño profesional y compromiso de responsabilidad social. Al estudiar la influencia de los perfiles docentes en el uso y preferencia de dinámicas de trabajo en grupo con alumnos universitarios, Marín-Garcia y Conchado-Peiró (2012) señalan que, entre los factores relevantes se encuentra: el área de conocimiento, la experiencia docente y los conocimientos y opinión que se tiene de las tecnologías activas. Sánchez (2012), utilizando la presentación de un problema integrador de contenido para ser resuelto a lo largo del semestre, el cual es dividido en problemas más específicos relacionados entre sí y utilizando actividades de aprendizaje como la exploración y transferencia de contenidos, logró evidenciar un progreso en el rendimiento académico y en el pensamiento crítico en estudiantes de Física.

Evaluación: Los módulos se inician con la indagación acerca de los conocimientos previos o preconcepciones de los estudiantes en calidad de evaluación diagnóstica (Anexo $N^{\circ} 1$ : ¿Qué sabes sobre las drogas?). Se enfatiza en la evaluación formativa en relación a los ámbitos cognitivo y valórico durante el desarrollo de las clases a fin de captar el interés de los estudiantes, para lo cual cada actividad es motivo de reflexión y diálogo en las distintas instancias consideradas (Anexo $\mathrm{N}^{\circ} 1$. Actividad $\mathrm{N}^{\circ} 2$ : Reflexionar: a); b); c)., finalizando cada módulo con una evaluación de carácter metacogntivo (Reflexiona sobre tu aprendizaje). Se realiza evaluación sumativa utilizando diversos procedimientos evaluativos tanto cualitativos como cuantitativos (Anexo $\mathrm{N}^{\circ}$ 2: Pauta para evaluar el debate). Las modalidades de evaluación utilizadas, son totalmente coherentes con el enfoque constructivista del aprendizaje que orienta los Módulos Alternativos de Aprendizaje.

\section{RESULTADOS}

Los Módulos de Aprendizaje Alternativos han ocupado un lugar central en el desarrollo del Proyecto FONDECYT 1100378 (Osses, 2010). En efecto, durante el Primer Semestre de 2010, profesores de las Comunas e investigadores, elaboraron el Módulo de Tercer Año y se piloteó el Segundo Semestre con los alumnos de este curso en las cuatro Comuna involucradas. El Segundo Semestre del año 2011, sin que fuera necesario hacerle modificaciones, de acuerdo al resultado del pilotaje, se aplicó este Módulo a los alumnos de Tercer Año. Además, el Primer Semestre de 2011, profesores de las Comunas e investigadores elaboraron el módulo de Cuarto Año y se piloteó el Segundo Semestre del mismo año en las cuatro comunas; después de realizar las modificaciones sugeridas en el pilotaje, fue aplicado a los alumnos de Cuarto Año el Segundo Semestre del año 2012.

En la Tabla 1, se muestra el resultado de la aplicación de los Módulos Alternativos, en términos del rendimiento promedio de los estudiantes de Tercer Año en Biología por semestre el año 2011. Las calificaciones obtenidas el Primer Semestre, corresponden al desarrollo de las clases sin Módulo, apoyadas en su mayor parte por el Texto de Biología aprobado por el Ministerio de Educación, en cambio, la notas del Segundo Semestre corresponden a clases realizadas con el apoyo mayoritario de los Módulos Alternativos de Aprendizaje.

Tabla 1: Promedio calificaciones semestrales de alumnos de Tercer Año de las 4 Comunas seleccionadas.

\begin{tabular}{|l|c|c|c|c|}
\cline { 2 - 5 } \multicolumn{1}{c|}{} & Carahue & Collipulli & Lonquimay & Puerto Saavedra \\
\cline { 2 - 5 } \multicolumn{1}{c|}{} & $(\mathrm{N}=33)$ & $(\mathrm{N}=25)$ & $(\mathrm{N}=20)$ & $(\mathrm{N}=24)$ \\
\hline I Semestre (sin módulo) & 5.51 & 5.64 & 5.80 & 4.35 \\
\hline II Semestre (con modulo) & 5.80 & 6.39 & 6.57 & 5.13 \\
\hline Diferencia & 0.29 & 0.75 & 0.77 & 0.78 \\
\hline
\end{tabular}

En la Tabla 1 se puede observar que, en todas las Comunas, el promedio de calificaciones del Segundo Semestre fue superior al primero, en diferente grado. Si bien es cierto, no es posible atribuir exclusivamente al Módulo el cambio en las calificaciones del Segundo Semestre a partir de una aproximación cualitativa, sin embargo, a través de ella, se puede verificar que existió un avance en los estudiantes entre ambos 
semestres; la cuantificación precisa del efecto correspondiente a la estrategia modular debería medirse a través de métodos estadísticos, lo que sobrepasa el alcance de este artículo. La técnica cualitativa a utilizar en este caso - grupos focales cuyos textos originales fueron analizados mediante el programa atlas $\mathrm{Ti}$ podría considerarse una primera aproximación a la evaluación de los resultados. En este contexto, a continuación, se presenta algunas citas provenientes de los grupos focales realizados con los estudiantes de Tercer Año y con los profesores de Biología de las Comunas, al finalizar el trabajo con los Módulos el año 2011.

\section{Grupo Focal Profesores}

Del análisis de los grupos focales realizados por los profesores, surgen las categorías: estrategias de aprendizaje; estrategias didácticas cognitivo afectivas y valores desarrollados por los alumnos durante el módulo. A continuación, se menciona las subcategorías que emergen de cada categoría y la cita que respalda a cada una de ellas.

Estrategias de aprendizaje. En esta categoría aparecen las subcategorías: fundamentar lo aprendido e investigar en distintas fuentes.

"Poder organizar su fundamentación porque es distinto que a veces ellos investigan a que ellos escriban cosas o copian y pegan".

"Investigar más en distintas fuentes y en internet, por supuesto".

Estrategias didácticas cognitivo-afectivas utilizadas por el docente, categoría en que se destacan las subcategorías: desarrollo de valores en los alumnos y fomento del respeto.

"Para mí lo innovador de este módulo fue la parte valórica y a los niños les gustó mucho eso; yo en un principio se los mostré y les dije que el aprendizaje no solamente tenía una (parte) cognitiva sino que una importante parte de su aprendizaje tenía que ver con los valores; ahí vimos por qué y qué valores debieran estar implícitos siempre cuando nosotros trabajamos con el módulo y fuimos viendo, cuando hubo tiempo, fuimos recordando eso en el grupo con ellos, ellos lo fueron considerando..."

"El saber escuchar, aprender a escuchar porque ellos no se escuchan, ellos hablan y gritan todos de una vez y no se saben escuchar y también valorar lo que está diciendo el otro, eso es super importante".

Valores desarrollados por los alumnos durante el módulo, categoría que incluye dos subcategorías: la responsabilidad y la solidaridad.

"La responsabilidad, a través del trabajo principalmente individual, también grupal; como hubo interrupciones, (paros y tomas), muchas veces ellos se llevaban el módulo y tenían que hacer aquella actividad y luego, la clase siguiente que nos viéramos lo contábamos y llegaban todos con sus actividades y su trabajo hecho":

"La solidaridad porque en mi comuna no todos tienen internet y computador.nada de eso; ellos se las arreglaban de manera que los que podían y tenían su equipo, invitaban a los demás a trabajar y yo lo encontré bien interesante".

\section{Grupo Focal Alumnos}

Del análisis de la información recopilada a partir de los grupos focales realizados por los estudiantes, surgen las categorías: estrategias de aprendizaje a desarrollar, aprendizaje generado a partir de la guía sobre drogas, evaluación del trabajo, opinión sobre las actividades, valores desarrollados durante el módulo, organización del trabajo grupal.

Estrategias de aprendizaje a desarrollar, categoría que incluye las subcategorías: resumir y concentrarse.

"La concentración, porque yo empiezo a leer y empiezo a conversar"

"Resumir el texto, sacar lo más importante"

Aprendizaje generado a partir de la guía sobre drogas. Esta categoría incluye las subcategorías: tipos de drogas y consecuencias de las drogas.

"Que en las drogas hay drogas lícitas e ilícitas; las lícitas corresponden a lo que es legal como medicamentos y las ilícitas que pudieran ser la marihuana o la cocaína" 
Evaluación del trabajo. De esta categoría emergió la subcategoría: autoevaluación.

"Sí, nosotros evaluábamos nuestro trabajo porque la profesora pasaba una hoja y nosotros teníamos que escribir nuestro nombre y cada uno evaluar a su compañero, cómo lo vio, cómo se expresó, cómo lo vio en la disertación".

Opinión sobre las actividades, categoría en la que se identifica la subcategoría: debate.

"A mí me gusta debatir de cómo conversar sobre un temas y dar tu opinión y no sé, encontré que fue interesante porque aparte de uno aprender más, también le ayuda a crecer de manera intelectual porque uno tiene que prepararse y para prepararse tiene que investigar, tiene que buscar información, entender la información para poder después defender su postura, para mí fue bueno y fue como un crecimiento personal"

Organización del trabajo grupal, categoría en la que se identificó dos subcategorías: monitorear avances y dividir el trabajo grupal.

"Nos preocupábamos de que todos fueran avanzando cada día más".

"Primero nos organizábamos y luego nos distribuíamos el trabajo y después investigábamos".

Valores desarrollados durante el Módulo, categoría desde la cual emergen las subcategorías: respeto, compañerismo y responsabilidad.

"Igual tenemos que escuchar las opiniones de los demás porque hay varias personas que tienen diferentes opiniones o pueden dar una nueva idea para hacer el trabajo, para poder ordenarlo, igual aprender a escuchar es una práctica que se hizo al hacer el trabajo y organizarlo".

"El compañerismo que nos damos mutuamente"

"La responsabilidad, porque dividíamos el trabajo y a cada uno le tocaba su parte, teníamos que responder"

\section{DISCUSIÓN DE LOS RESULTADOS}

Al finalizar la aplicación de los Módulos Alternativos, los estudiantes participantes muestran un alza en sus calificaciones. Es probable que, parte de ese efecto se deba a la estrategia didáctica utilizada. Sin embargo, si se desea cuantificar con precisión la magnitud de tal efecto, sería necesario aplicar a los resultados tests estadísticos que están fuera del alcance de este artículo (Hernández, Fernández y Baptista, 2010), cuya naturaleza es cualitativa.

Con respecto al rendimiento, los profesores señalan que los estudiantes mostraron algunos avances en el ámbito cognitivo, mencionando, por ejemplo, que aprendieron a fundamentar sus afirmaciones pasando de la simple opinión al juicio. Es probable que esto se haya visto favorecido por las estrategias de enseñanza utilizadas (Díaz-Barriga y Hernández, 2002), entre otras, la exigencia de realizar investigación, para la cual, los estudiantes utilizaron fuentes variadas de información y, además, la realización de actividades como el debate que, necesariamente, requirió de los estudiantes una preparación profunda a objeto de fundamentar su posición.

Los estudiantes, en cuanto a su rendimiento, por una parte, muestran progreso en la toma de conciencia acerca de sus propios procesos cognitivos (Bixio, 2001), manifestado en el reconocimiento de las estrategias de aprendizaje que deben seguir desarrollando, la valorización de la autoevaluación como herramienta para detectar en forma equilibrada fortalezas y debilidades, y el reconocimiento de algunos aspectos de la temática del módulo que ellos declaran haber aprendido. Por otra parte, dan cuenta de la organización del trabajo grupal que llevaron a la práctica, caracterizada por la división del trabajo y el monitoreo constante que realizaron sobre los avances logrados, vivenciando de este modo, la autorregulación de sus procesos cognitivos (Soto, 2002).

Esto es confirmado por Shulman (2005) quien, en estudios tendientes a lograr aprendizajes significativos en los estudiantes, enfatiza en la creatividad, comprensión, razonamiento y reflexión, en una palabra, en el ejercicio de la metacognición. Del mismo modo, estudios recientes en China (Deng et al., 2011) demuestran que la metacognición, en estudiantes de nivel preuniversitario, está significativamente asociada con su comprensión conceptual en Química, lo que favorece notoriamente el aprendizaje y coincide con los resultados obtenidos por Hongxia y Zhibo (2010) en relación a la importancia de la metacognición en clases de escritura en China y otros países, señalando que los profesores deben enfrentar el gran desafío de desarrollar la autonomía en sus estudiantes, a fin de que continúen aprendiendo a lo largo de su vida. 
La total coincidencia entre profesores y estudiantes respecto del desarrollo de valores a través del módulo, da cuenta de que el efecto de su aplicación se extendió más allá de lo meramente cognitivo, abarcando simultáneamente el ámbito afectivo (Román, 2005).

\section{HALLAZGOS}

Los hallazgos se plantean a partir de los resultados de la aplicación de técnicas cualitativas de validación de los grupos focales realizados con profesores y estudiantes, específicamente, triangulación de resultados y saturación de datos, sin desconocer que existe información adicional proporcionada por instrumentos cuantitativos (Osses, 2013), que refuerza los resultados cualitativos obtenidos pero que, por la naturaleza cualitativa de este artículo no se les ha mencionado en detalle.

Profesores y estudiantes de Tercero y Cuarto Año Medio de los Liceos Municipalizados de las Comunas de Collipulli, Lonquimay, Carahue y Puerto Saavedra, participaron en el Proyecto FONDECYT 1100378, en el cual se utilizó una metodología de trabajo centrada en Módulos de Aprendizaje Alternativos, orientados a mejorar en los estudiantes el aprendizaje en Biología.

En este proceso, se ha detectado profundos cambios en el rol del docente que, de transmisor se ha transformado en mediador, lo que se ha traducido en cambios positivos en el aula, ya que los estudiantes han utilizado sus conocimientos previos, han investigado y compartido sus hallazgos con los compañeros de grupo y con el curso, en general. Además, al ir construyendo su propio conocimiento, al reflexionar y tomar conciencia de su aprendizaje, logros y progresos, han desarrollado procesos metacognitivos (Hugo et al., 2009). Del mismo modo, se ha fortalecido la interacción profesor - alumno, gracias a la constante invitación del docente a descubrir y poner en práctica sus propias potencialidades por parte de los estudiantes y, a la vez, éstos con sus sugerencias, han iluminado al profesor respecto de la selección de actividades en futuras situaciones de aprendizaje.

Como resultado de la aplicación de grupos focales a estudiantes y profesores, éstos últimos han expresado que, a través de los Módulos Alternativos, los estudiantes han desarrollado capacidades y valores. A partir de ello se infiere que esta estrategia didáctica promueve la formación integral de los educandos abarcando los ámbitos cognitivo y afectivo.

De todo lo anterior se desprende que, es preciso continuar enseñando a los estudiantes a desarrollar procesos de reflexión metacognitiva, porque aportan al desarrollo del pensamiento, al logro de habilidades cognitivas superiores y a la construcción de su propio conocimiento, en tanto les permite planear, controlar y evaluar responsablemente el desarrollo de las tareas a realizar (Day, 2006). Del mismo modo, es necesario continuar enseñándoles a desarrollar valores tendientes a humanizar nuestra sociedad, superando la corriente positivista a ultranza imperante actualmente (Román, 2005).

En consecuencia, es ineludible la tarea de seguir profundizando en la elaboración y puesta en práctica de Módulos Alternativos de Aprendizaje, quedando como tarea pendiente la ampliación de su radio de acción hacia otras temáticas y niveles educativos, su enriquecimiento con nuevas estrategias didácticas y la aproximación cuantitativa a la evaluación de sus resultados, a fin de avanzar hacia la formación de estudiantes motivados, creativos, autónomos, críticos y responsables, es decir, avanzar en dirección a una educación de calidad.

\section{AGRADECIMIENTOS}

Agradecemos a CONICYT el valioso aporte que ha significado para nuestro equipo, el financiamiento del Proyecto FONDECYT Regular 1100378, en el cual se basa el presente artículo. 


\section{ANEXO No 1}

“MÓDULO DE APRENDIZAJE ALTERNATIVO PARA TERCER AÑO DE EDUCACIÓN MEDIA . BIOLOGÍA E HIGIENE NERVIOSA

TEMA: DROGAS Y TOXICOMANÏA

\section{OBJETIVOS:}

- Conocer y comprender qué son las drogas, cuáles son y sus efectos en el organismo.

- Relacionar nueva información con conocimientos previos.

- Fortalecer vínculos de apoyo e interrelación entre pares.

- Tomar conciencia de sus propios procesos cognitivos.

- Desarrollar la capacidad de autorregular su aprendizaje.

- Aplicar los conocimientos adquiridos a distintos contextos.

- Desarrollar valores tales como la responsabilidad, el respeto y la participación.

\section{PRESENTACIÓN}

Estimado(a) estudiante:

Con mucho afecto ponemos en tus manos estos Módulos de Aprendizaje en Biología, para que, a través de ellos, tengas la oportunidad de conocer un tema vivencial, como es Biología e Higiene Nerviosa y sus implicancias, como asimismo, reflexionar y dialogar sobre ellas con tus compañeros (as) y personas de la comunidad en que estás inserto, especialmente, con tu familia.

A través del módulo pretendemos que construyas conocimientos, puedas compartir sentimientos y creencias con respecto al alcohol y a otras drogas y dialogar sobre el tema, teniendo la oportunidad de aprender a tomar decisiones responsables.

Durante el proceso de aprendizaje en Biología, tú serás el protagonista, es decir, lo que aprendas dependerá de ti, de tu entusiasmo, responsabilidad y creatividad. Tu profesor(a) será un guía, de modo que podrás, con tranquilidad, acoger las actividades sugeridas y llevarlas a la práctica, proponer actividades diferentes $y$, en general, elegir entre alternativas en variados temas.

Es preciso señalar que, los módulos no se agotan en el mero conocimiento científico. En efecto, a lo largo de su desarrollo, se da gran importancia al aspecto de formación, especialmente en responsabilidad personal y social, lo cual se refleja, claramente, en las Guías de Aprendizaje.

Esperamos que el trabajo con este material, signifique para ti una oportunidad de profundizar en temas de enorme trascendencia social y, a través del desarrollo de los ámbitos cognitivo y valórico, contribuya a tu formación integral como persona comprometida con la armonía con tu medio natural y sociocultural.

\section{¿QUÉ SABES SOBRE LAS DROGAS?}

Te invitamos a recordar y reflexionar sobre los conocimientos adquiridos en tu familia y entorno. Te proponemos responder las preguntas que se plantean a continuación, poniendo énfasis en el fundamento de cada una de ellas. Ten presente que tus conocimientos previos permitirán construir conocimientos nuevos sobre una base más sólida, forjar un estilo de vida y adaptarte sanamente a cada una de las áreas de la vida cotidiana.

Responde las siguientes afirmaciones, colocando frente a cada afirmación Verdadero (V) o Falso (F) y justifica las respuestas falsas donde corresponda. 


\begin{tabular}{|l|l|l|l|}
\hline Preguntas & V & F & Justifica las Falsas. \\
\hline 1.- El consumo de drogas es sólo un problema de los jóvenes. & & & \\
\hline 2.- Las personas consumen drogas sólo cuando tienen problemas. & & & \\
\hline 3.- Los no fumadores no sufren los efectos del tabaco. & & & \\
\hline 4.-Todas las drogas producen los mismos efectos. & & & \\
\hline $\begin{array}{l}\text { 5.- Es importante que los niños prueben bebidas alcohólicas en } \\
\text { compañía de sus familiares. }\end{array}$ & & & \\
\hline $\begin{array}{l}\text { 6.- En relación a las drogas, existen los que consumen y los que son } \\
\text { dependientes. }\end{array}$ & & & \\
\hline $\begin{array}{l}\text { 7.- Si una droga es natural, es decir, no procesada, es menos dañina } \\
\text { que una sintética. }\end{array}$ & & & \\
\hline $\begin{array}{l}\text { 8.- Si no se bebe alcohol en una fiesta es, generalmente, una fiesta } \\
\text { "fome" }\end{array}$ & & & \\
\hline $\begin{array}{l}\text { 9.- Si vendo o regalo drogas a mis amigos o conocidos, no cometo } \\
\text { ningún delito. }\end{array}$ & & & \\
\hline $\begin{array}{l}\text { 10.- Estar informado acerca de las drogas, ayuda a optar por un estilo } \\
\text { de vida saludable. }\end{array}$ & & & \\
\hline 11.- El alcohol y el tabaco producen enfermedades. & & \\
\hline
\end{tabular}

\section{GUÍA DE APRENDIZAJE}

\section{¡APRENDAMOS SOBRE LAS DROGAS!}

\section{ACTIVIDAD N 1: "LEER COMPRENSIVAMENTE"}

\section{Materiales}

Historia de vida, Anexos $N^{\circ}$ 1, 2, 3, 4. Texto del Alumno. Aprendamos a Aprender. Biología Humana. Higiene Nerviosa. Módulo para el estudiante 3. 2011. (González, M; Manríquez, M, Osses, S. y otros recursos incluyendo los computacionales.

a) Se agrupan de a cuatro estudiantes y leen en silencio el caso dado.

b) Subrayan las principales ideas y hacen inferencias sobre el contenido del material utilizado.

c) Comentan y discuten las posibles causas que conducen al consumo de drogas.

\section{ACTIVIDAD N²: "REFLEXIONAR"}

a) ¿Cuáles decisiones, que tomó o dejó de tomar el protagonista de la historia de vida en el pasado, podrían haberle evitado llegar al problema?

b) ¿Qué decisión le ayudaría a que le fuera bien en su vida?

c) ¿Qué factores influyen que, en este caso, haya riesgo de abuso de alcohol y drogas?

d) Plenario: cada grupo presenta sus conclusiones, se hace una pausa para detenerse en los desacuerdos buscando los conflictos implícitos y, posteriormente, las soluciones.

e) Cierre: Al finalizar la actividad, se analiza y se hace una síntesis de los factores de riesgo que pueden llevar al consumo de drogas.

\section{ACTIVIDAD No 3: "COMPARAR, FORMULAR HIPÓTESIS, INTERPRETAR Y PLANTEAR} CONCLUSIONES"

a) Forman grupos de cuatro estudiantes e investigan en internet acerca de los estudios de CONACE sobre el consumo de drogas en escolares chilenos.

b) Seleccionan un estudio reciente y otro de años anteriores, con el fin de comparar tablas de datos y gráficos y formulan hipótesis que expliquen la distribución y evolución del consumo de drogas. 
c) Interpretan y comparan datos, emiten juicios críticos respecto a la magnitud del fenómeno.

d) Determinan cuáles son los factores de riesgo más importantes que inciden en el consumo de drogas en la población escolar.

e) Concluyen sobre la base de los datos analizados

f) Hacen puesta en común y socializan sus conclusiones.

\section{ACTIVIDAD N 4: "INVESTIGAR, COMUNICAR, DEBATIR Y VALORAR"}

El Debate consiste en un intercambio informal de ideas e información sobre un tema que se presta a la controversia, realizado por un grupo, bajo la conducción dinámica y estimulante de un coordinador o moderador.

En grupos de 6 estudiantes investigan temas contrapuestos, entregados por el profesor, que están relacionados con el efecto de las drogas lícitas e ilícitas en el cuerpo humano.

Cada grupo investiga sobre la postura que le corresponderá defender y es responsable de:

a) Estudiar lo que se le propone y ampliar la información sobre el tema.

b) Separar la información verificable de las opiniones personales.

c) Disentir de lo que considere erróneo o falso.

d) Seleccionar y diferenciar lo relevante de lo secundario.

e) Sacar conclusiones propias. Adicionalmente, se tiene que preparar los materiales de apoyo (cuadros estadísticos, mapas conceptuales, grabaciones, etc.)

f) Deben llegar a la sala de clases, bien preparados. Es importante recurrir a argumentos lógicos, para defender un punto de vista o una idea. El tipo de lenguaje debe ser argumentativo.

g) Cada grupo deberá elegir un representante que comenzará el debate. Luego, todos los participantes deben debatir según el turno que le dé el moderador.

h) El debate durará 20 minutos. Cada expositor dispondrá de tres minutos para cada una de sus intervenciones. La intervención puede ser interrumpida, siempre y cuando el expositor lo permita y se le solicite respetuosamente.

i) Terminado el debate, el Secretario tratará de que la asamblea llegue a las conclusiones.

j) Como actividad de cierre, realizan difusión del tema a través de un tríptico preparado por ellos a otros cursos, colegio, etc.

\section{Actividad de Término del Módulo}

\section{REFLEXIONA SOBRE TU APRENDIZAJE}

Nombre

Fecha:

Establecimiento:

Curso:

\section{¿QUÉ APRENDI? ¿POR QUÉ CREO QUE LO APRENDÍ?}

\section{¿CÓMO LO APRENDÍ?¿TUVE OTRAS ALTERNATIVAS PARA APRENDER?¿POR QUÉ SEGUÍ ESE CAMINO?}




\section{ANEXO No 2}

\section{PAUTA PARA EVALUAR EL DEBATE}

Nombre:

Fecha:

\begin{tabular}{|l|l|l|l|l|}
\hline \multirow{2}{*}{ Aspectos generales del tema en discusión } & \multicolumn{2}{|c|}{ Grupo A } & \multicolumn{2}{c|}{ Grupo B } \\
\hline & SI & NO & SI & NO \\
\hline El tema interesa a la mayoría & & & & \\
\hline Plantean claramente los objetivos & & & & \\
\hline Existe investigación previa del tema & & & & \\
\hline Se analiza a fondo el tema tratado & & & & \\
\hline Se respetan los tiempos & & & & \\
\hline Se argumenta en forma lógica & & & & \\
\hline Se muestra dominio del tema & & & & \\
\hline Se manifiesta capacidad de síntesis o resumen & & & & \\
\hline El lenguaje utilizado por la mayoría de los \\
participantes ha sido:
\end{tabular}

\begin{tabular}{|l|l|l|l|l|}
\hline Respecto al moderador & SI & NO & SI & NO \\
\hline Domina el tema & & & & \\
\hline Organiza bien las intervenciones & & & & \\
\hline Clarifica conceptos cuando es necesario & & & & \\
\hline Encauza el tema en discusión & & & & \\
\hline Favorece la intervención de todos los participantes & & & & \\
\hline Se muestra imparcial ante las diversas posiciones & & & & \\
\hline Extrae conclusiones & & & & \\
\hline $\begin{array}{l}\text { Realiza una síntesis final basado en las } \\
\text { conclusiones }\end{array}$ & & & & \\
\hline
\end{tabular}




\section{REFERENCIAS}

Álvarez-Gayou, J., Cómo hacer investigación cualitativa. Paidós Educador , México,D.F. (2005).

Álvarez, F., Rodríguez-Pérez, J., Sanz-Ablanedo, E., y Fernández - Martínez, M., Aprender Enseñando: Elaboración de Materiales Didácticos que facilitan el Aprendizaje Autónomo. Formación Universitaria, 1(6), 19-28. (2008).

Bautista, N., Proceso de la investigación cualitativa. El Manual Moderno, S.A, de CV, Bogotá, Colombia (2011).

Bixio, C., Contenidos procedimentales. Los procedimientos: su enseñanza, aprendizaje y evaluación. Rosario: Homo sapiens, Santa fe, Argentina (2001).

Bogumil, F., y Abad, F., Mejoras del Aprendizaje y del Rendimiento de Grupos Reducidos de Estudiantes en una Asignatura de Proyectos de Ingeniería. Formación Universitaria, 1(3) 13-20 (2008).

Correa, C., Currículo dialógico, sistémico e interdisciplinar. Subjetividad y desarrollo humano, Cooperativa Editorial Magisterio, Bogotá, Colombia (2004).

Day, C., Pasión por enseñar. La identidad personal y profesional del docente y sus valores, Narcea S.A, Madrid, España (2006).

Deng, F., Chen, W., Sing,C., y Qian,Y., Constructivist-oriented Data-logging Activities in Chinese Chemistry Classroom: Enhancing Students' Conceptual Understanding and Their Metacognition. The Asia-Pacific Education Researcher, 20(2),207-221(2011).

Díaz -Barriga, F., y Hernández, G., Estrategias docentes para un aprendizaje significativo. Mc Graw-Hill, México (2002).

Frith,C., The role of metacognition in human social interactions. Philosophical Transactions B, 367(1599)2213-2223 (2012).

Hernández,R., Fernández,C., y Baptista,P., Metodología de la investigación. Quinta edición. Mac Graw Hill, México (2010).

Hugo, E., Osses, S., y Pacheco, M., Aprendamos a aprender: Biología humana y salud Microorganismos y sistemas de defensa. Módulo para Cuarto Año de Enseñanza Media. Proyecto Fondecyt Regular № 1070256 (2009).

Hennink,M., Hutter, I., y Bailey, A., Qualitative Research Methods. SAGE Publications, London, England (2010).

Hongxia, Y., y Zhibo, H., A Review of the Empirical Research on Metacognition in ESL/EFL Writing, International Forum of Teaching and Studies, 6(2) 34-40 (2010).

Jaramillo, S., Dimensión metacognitiva en módulos de aprendizaje en Biología. Aproximación a un proceso de validación. Tesis para optar al Grado de Magíster en Educación Ambiental. Revista Investigaciones en Educación: 9(1), 127-143. (2009).

Mahmud, M., y Gutiérrez, O., Estrategia de Enseñanza Basada en el Cambio Conceptual para la Transformación de Ideas Previas en el Aprendizaje de las Ciencias. Formación Universitaria, 3(1) 11-20 (2010).

Marcelo, C., Aprender a enseñar para la sociedad del conocimiento. Investigaciones en Educación, vol.3, 11-33. (2003).

Marín-García, J., y Conchado-Peiró, A., Influencia de los Perfiles Docentes en el Uso y Preferencia de Dinámicas de Trabajo en Grupo con Alumnos Universitarios, Formación Universitaria 5(5) 3-14. (2012).

Mateos, M., Metacognición y educación, Grupo Editor Aique, Buenos Aires, Argentina (2001).

Menchén, F., La Creatividad en el aula. Perspectiva teórico - práctica. Conocimiento S.A, Santiago, Chile (2008).

Miles, M., y Huberman, M., Qualitative data analysis: An expanded sourcebook, Thousand Oaks, CA: Sage. (1994).

Ontoria, A., Gómez, J., y Molina, A., Potenciar la capacidad de aprender a aprender, Alfaomega Grupo Editor, México DF, México (2003).

Osses, S., Hacia un nuevo enfoque en la Enseñanza de las Ciencias. Convergencia entre modelo constructivista y dimensión Ciencia/ Tecnología/ Sociedad. Proyecto Fondecyt Regular № 1970258. (1997). 
Osses, S., Hacia un nuevo enfoque en la Enseñanza de las Ciencias, Convergencia entre modelo constructivista y dimensión Ciencia/ Tecnología / Sociedad. Revista Investigaciones en Educación. Vol. 2:79-96 (2002).

Osses, S., Hacia la autonomía profesional del docente. El profesor como investigador en el aula. Proyecto Fondecyt Regular No 1030278 (2003).

Osses, S., Proyecto FONDECYT Regular No 1100378 (2010).

Osses, S., Informe Final Proyecto FONDECYT Regular No 1100378 (2013)

Osuna, C., y Luna, E., Valores Éticos en la Formación Universitaria de las Áreas de Ciencias Naturales e Ingeniería y Tecnología, en el Contexto de la Sociedad del Conocimiento, Formación Universitaria, 4(5) 2936 (2011).

Pérez, G., Investigación cualitativa. Retos, interrogantes y métodos, La Muralla, España (1994).

Pimienta, J., Estrategias para aprender a aprender, Pearson Educación de México S.A. de C.V (2008).

Román, M., Capacidades y valores como objetivos en la sociedad del conocimiento. Perspectiva didáctica. Arrayán Editores S.A., Santiago, Chile (2005).

Robledo, J., Observación Participante: el acceso al campo, Nure Investigación, 40, 2009.

Sánchez, I., Evaluación de una Renovación Metodológica para un Aprendizaje Significativo de la Física, Formación Universitaria,5 (5) 51-65 (2012).

Shulman, L., Conocimiento y enseñanza: Fundamentos de la nueva Reforma. Revista de Curriculum y formación del profesorado: 92(2)11-26 (2005).

Soto,C., Metacognición, Cambio conceptual y enseñanza de las Ciencias, Cooperativa Editorial Magisterio, Bogotá, Colombia (2002). 